\title{
THEORETICAL PROBLEMS OF STELLAR MAGNETISM
}

\author{
LYMAN SPITZER, JR. \\ Princeton University Observatory, Princeton, N.J., U.S.A.
}

\begin{abstract}
ABSTRAGT
Relatively complete summaries of recent theoretical work in the field of stellar magnetism have been given by Cowling [1] and Elsasser [2, 3,4]. For this reason the present survey does not aim at completeness, but is devoted instead to the following three outstanding problems in the field: ( 1 ) the formation of stars in the presence of an interstellar magnetic field; (2) the influence of convection. currents on a stellar magnetic field; (3) the nature of magnetic variable stars. Study of these problems illustrates the type of difficulties encountered in theories of stellar magnetism.
\end{abstract}

\section{STAR FORMATION IN A MAGNETIZED GAS}

The observed polarization of starlight is probably caused by an interstellar magnetic field. The field strength, $B$, required to explain the observed polarization is about $10^{-5}$ gauss, if the Davis-Greenstein [5] theory of paramagnetic relaxation is accepted. However, a substantially lower field may be sufficient. Gorter suggested some time ago that ferromagnetic relaxation would be a much more dissipative influence than paramagnetic relaxation, provided we can assume, following Spitzer and Tukey [6], that an appreciable fraction of the grains are ferromagnetic. This suggestion has been analysed by $\mathrm{J}$. Henry, who finds that a field of $10^{-7}$ gauss should suffice. However, $\mathrm{B}$ cannot be much less than $2 \times 10^{-6}$ gauss if cosmic rays are assumed to be confined within the Galaxy by magnetic fields.

Another hypothesis which is now widely accepted is that stars are forming from the interstellar gas. Accretion, as compared to direct condensation, may also be important, but in either case we may assume that the interstellar material is being brought together to form the young $\mathrm{O}, \mathrm{B}$ and $\mathrm{A}$ stars of population type I.

It is therefore important to investigate the effects of a magnetic field on the contraction of an interstellar cloud. If the flux through the star is 169 
assumed constant, it is readily shown that the ratio of gravitational to magnetic forces remains constant during the contraction. If the condensation is large enough and dense enough to permit contraction to begin, magnetic forces will not prevent further contraction.

However, while condensations can develop, the minimum mass that can condense is large, greater than $10^{3}$ suns for a field of $2 \times 10^{-6}$ gauss or more. If the flux through the material remains constant there seems no way in which smaller stars can be formed from a big condensation, since the ratio of gravitational to magnetic pressure decreases as the mass decreases. In the case of the sun, for example, if the magnetic field were $2 \times 1^{-6}$ gauss when the density of presolar matter was 10 atoms $/ \mathrm{cm}^{3}$, corresponding to a typical $\mathrm{H}_{\mathrm{I}}$ cloud, the present magnetic field at the center of the sun would be ${ }^{1} 0^{11}$ gauss, corresponding to a magnetic pressure about $10^{3}$ times as great as the material pressure there. For the young stars of types $\mathrm{O}$ and $\mathrm{B}$ the discrepancy is almost as serious. If the stars are to form from interstellar clouds, either the initial field must be much less than $2 \times 10^{-6}$ gauss, or there must be some way in which the magnetic flux through the material can be decreased.

Fortunately a powerful mechanism is available for reducing the flux through a contracting protostar. In a partially ionized gas the lines of force are attached to the electrons and positive ions, not to the neutral particles. The outwards force resulting from magnetic pressure is applied to the charged particles, and from these is transmitted to the neutral particles by frictional forces. These frictional forces can arise only from an outward drift of the electrons and ions relative to the neutral particles. The fewer electrons and ions present, the more rapid this outwards drift must be to transmit the same force. Since the relative ionization probably falls to a very low value in a protostar, owing to the extinction of ultraviolet radiation by the grains, this relative drift can be rapid. As a result, the lines of force, which have been stretched by the initial condensation of the cloud, pull their way out of the cloud, bringing the charged particles with them, and leaving a relatively low flux through the contracting cloud. Each line of force retains its identity in this process, but the magnetic energy decreases as the lines of force straighten, the energy being dissipated by the frictional force between the charged particles and the neutral atoms. The dissipation of energy occurs by the same process which Piddington [7] and Cowling (1955, informal communication) have proposed for the heating of solar flares.

A detailed analysis of this process, given by Mestel and Spitzer [8], will be summarized here. Let $F$ be the outwards force on the electrons and 
positive ions in a cubic centimeter. Since $F$ is produced by the gradient of the magnetic pressure, we have approximately, in c.g.s. units,

$$
F=\frac{B^{2}}{8 \pi R}
$$

where $R$ is the linear dimension of the cloud. In a quasi-steady state, the force $F$ will be balanced by the frictional force between positive ions and neutral atoms. If $n_{i}$ and $n_{H}$ are the particle densities of positive ions and neutral hyorogen atoms, respectively, and if $v_{D}$ is the relative drift between them, the number of collisions $/ \mathrm{cm}^{3}$ per sec will be $n_{i} n_{H} \overline{\sigma v}$, where $v$ is the random relative velocity and $\sigma$ is the collision cross-section. The momentum transferred at each collision will be approximately $m_{H} v_{D}$. Hence

$$
F=n_{i} n_{H} \overline{\sigma v} m_{H} v_{D}
$$

Equating (I) and (2), we find that

$$
v_{D}=\frac{B^{2}}{8 \pi R n_{i} n_{H} \overline{\sigma v} m_{H}} .
$$

Let us apply this result to a large cloud complex, some $30 \mathrm{pc}$ in diameter, containing about $5 \times \mathrm{IO}^{3}$ solar masses, that has contracted to a tenth of its original size, increasing $n_{H}$ to ${ }^{4} 0^{4} / \mathrm{cm}^{3}$, and $B$ to $2 \times 10^{-4}$ gauss. For the random mean velocity, $v$, we may take $10^{5} \mathrm{~cm} / \mathrm{sec}$, about the value obtained from the virial theorem, and let $\sigma$ equal ${ }_{10}^{-16} \mathrm{~cm}^{2}$. We find

$$
v_{D}=\frac{2 \cdot 2 \times \mathrm{IO}^{3}}{n_{i}} \frac{\mathrm{cm}}{\mathrm{sec}} \text {. }
$$

If the relative ionization were the same as in a normal $\mathrm{H}_{\mathrm{I}}$ cloud, with $n_{i} / n_{H}$ equal to $5 \times 10^{-4}, v_{D}$ would be about $4 \times \mathrm{IO}^{2} \mathrm{~cm} / \mathrm{sec}$, much too slow to achieve any separation.

However, $n_{i} / n_{H}$ must certainly be much reduced below its value in a normal interstellar cloud. The extinction through the cloud is increased by a factor of 100 in the contraction, and even in visible light will amount to some 20 magnitudes. Evidently $n_{i}$ will be determined primarily by the rate of recombination. Radiative recombination is not very effective, reducing $n_{i}$ by a factor of only $1 \mathrm{O}^{2}$ in $10^{5}$ years. Recombination generally takes place by means of other, more rapid, processes, and in the present instance dissociative recombination and recombination by collision with grains should be more rapid than radiative recombination by a factor of at least $\mathrm{IO}^{3}$. Thus $n_{i}$ should be well below $\mathrm{IO}^{-4} / \mathrm{cm}^{3}$, at the particular stage of contraction discussed above, and $v_{D}$ should be at least $\mathrm{I} \mathrm{km} / \mathrm{sec}$, even if $B$ is reduced to $10^{-5}$ gauss. 
Evidently the computed value of $v_{D}$ is so large that the assumption of a quasi-steady state, made in deriving Eq. (3), becomes incorrect. For very small values of $n_{i}$ the magnetic field nearly straightens itself out (or, if initial currents along the lines of force are present, at least nearly adjusts itself to a force-free condition) and the lines of force are then stationary as the gas contracts, the neutral atoms streaming by the electrons and positive ions; the small frictional force is offset by a slight, but roughly constant stretching of the lines of force.

We conclude that a magnetic field in interstellar space should not seriously interfere with the condensation of material to form new stars. One might expect some residual magnetic field to remain in a newly-born star, but it is difficult to predict the strength of such a field.

\section{INFLUENGE OF FLUID MOTIONS ON MAGNETIC FIELDS}

Internal motions are believed to be a characteristic of most stars-see the summary by Schwarzschild [9]. For stars later than type A a hydrogen convective zone is present at or below the surface, extending down in some stars by an appreciable fraction of the radius. For stars earlier than type $\mathbf{F}$ the central regions are assumed to be in convective equilibrium. There are probably very few stars which are in radiative equilibrium virtually throughout. In view of the tendency of lines of magnetic force to follow the material, fluid motions must have an intimate and powerful connexion with any magnetic properties of a star:

Much of the recent work on magnetic fields in moving fluids has been concerned with the dynamo theory of terrestrial magnetism. The objective of the research has been to show that fluid motions in a sphere can amplify an initial magnetic field and result in a finite external dipole field. The theoretical work, which has been summarized by Elsasser $[3,4]$ requires the assumption that the motions are not axially symmetric, since Cowling [10] has shown that a self-sustaining dynamo is impossible if the motions are axially symmetric. For reasons of mathematical simplicity these theories discuss the distortion of rather simple initial fields, and do not consider in detail the complicated magnetic effects produced by turbulence of all scales.

Actually, the interaction between turbulent motions and magnetic fields would seem to be a rather central problem of stellar magnetism. It is sometimes assumed that turbulence increases the effective resistivity of stellar material and accelerates the rate of decay of the external field. 
More precise information on this point would be very helpful. Certain general theorems on this subject will be presented below. As we shall see, one may show that in an idealized case, with axial symmetry, an arbitrary field of fluid motions has only a relatively minor effect on the decay time.

We shall treat first the case of an infinite fluid cylinder, with the restriction that all quantities are independent of the axial co-ordinate, $z$, except for an imposed electrostatic potential which equals $-E_{0} z$. This situation, which is mathematically simpler than the sphere, has been consided already by Sweet [11]. The fluid will be assumed incompressible and the resistivity, $\eta$, constant. The basic equation is Ohm's Law, which becomes

$$
E_{z}+v_{r} B_{\theta}-v_{\theta} B_{r}=\eta j_{z} \text {. }
$$

The $r$ and $\theta$ components of Ohm's Law need not be considered. If we introduce the vector potential $\mathbf{A}$, only the component $A_{z}$ appears in Eq. (5), and we have

$$
\frac{\partial A_{z}}{\partial t}+v_{r} \frac{\partial A_{z}}{\partial r}+v_{\theta} \frac{\partial A_{z}}{r \partial \theta}=E_{0}-\eta j_{z}
$$

We may express $j_{z}$ in terms of $A_{z}$ by the equation

$$
j_{z}=-\frac{\mathrm{I}}{4 \pi} \nabla^{2} A_{z} \text {. }
$$

The quantities $v_{z}$ and $B_{z}$ need not be zero, but do not enter into the determination of $A_{z}, B_{r}$ and $B_{\theta}$. A line of force in the $r, \theta$-plane is a line of constant $A_{z}$, and Eq. (6) describes the situation where arbitrary two-dimensional motions, in the $r, \theta$-plane, bend, twist and distort a magnetic field which is also in the $r, \theta$-plane.

Sweet [11] has demonstrated the important fact that when Eq. (6) is integrated over an area bounded by a line of force, the velocity terms cancel out. In a steady state $\partial A_{z} / \partial t$ vanishes, and we obtain

$$
\overline{\eta j_{z}}=E_{0},
$$

where the average is taken over the surface enclosed by a line of force. On the other hand, by Stokes' theorem,

$$
\frac{\eta}{4^{\pi}} \oint \mathbf{B} \cdot \mathbf{d} \mathbf{\lambda}=\eta \int j_{z} d S=E_{0} \int d S .
$$

Since the lines of force are made very crinkly by small-scale motions, the ratio of the length of the line of force to the area enclosed will be much increased. Hence the mean value of $B$, without regard to direction, will be decreased by the motions. The mean value of $B_{\theta}$ will be decreased even 
further. From this result Sweet concludes that the effective resistivity of the fluid is much increased by the presence of fluid velocities.

While it is true that the internal field is decreased by the fluid motions, we shall show that the external field is not affected. The velocity terms in Eq. (6) also cancel out when this equation is integrated over a surface area bounded by a stream line of the flow; this is evident on integrating by parts, and utilizing the equation of continuity. In particular these velocity terms cancel out if Eq. (6) is integrated over the entire cross-sectional area of the cylinder, since the normal velocity $v_{r}$ must vanish at the surface of the cylinder. Hence in the steady state, Eq. (8) is valid for the mean axial current in the cylinder, and if the total current is denoted by $I$, we have

$$
I=\frac{\pi R^{2} E_{0}}{\eta}
$$

where $R$ is the radius of the cylinder. Evidently the total current and the resultant external magnetic field are exactly the same as in the absence of fluid flow.

At first sight these two results, a weakened magnetic field inside and an unchanged field outside, seem contradictory. What actually happens is that in the bulk of the cylinder the magnetic field strength will be very low, and, by Eq. (7), $j_{z}$ will also be very low. The total current $I$ is concentrated in a thin layer close to the surface, where the effect of convection is much reduced. The ohmic dissipation $\eta j^{2}$ is increased, but this additional dissipation is offset by the work done on the magnetic field by the fluid velocities. It is readily shown that the decay time of a cylindrical field of this sort is almost entirely unaffected by the presence of fluid motions of the type assumed here.

This same analysis may be applied to a sphere, provided we assume that all quantities are symmetric about the axis. If we introduce co-ordinates $r, \theta$, and $\phi$, the equation for the component $A_{\phi}$ of the magnetic vector potential is again found to be independent of $v_{\phi}, A_{r}$ and $A_{\theta}$. Since there can obviously be no applied electrostatic potential in the $\phi$ direction, there is now no counterpart of the $E_{0}$ term in Eq. (6). The detailed analysis will be given elsewhere. Here we shall give simply the general result

$$
\frac{\partial}{\partial t}\left\{\int A_{\phi} \rho r \sin \theta d V\right\}=-\int \eta \rho j_{\phi} r \sin \theta d V,
$$

integrated over the entire volume, $V$, of the star; compressible flow has been assumed, and both the density, $\rho$, and the resistivity, $\eta$, must be assumed variable However, in a convective layer with $\gamma$ equal to $5 / 3, \rho$ varies as $T^{3 / 2}$; since $\eta$ varies as $T^{-3 / 2}$ in an ionized gas, the product $\eta \rho$ is 
constant, and may be taken out of the integrand. We shall here consider the extreme case in which the star is in convective equilibrium throughout. The product $\eta \rho$ is now constant throughout the star, and the integral on the right-hand side of Eq. (I I) may be expressed in terms of the external stellar dipole moment, $\mathscr{M}$. The dipole moment due to a current $j_{\phi}$ flowing across an area $d S$ around a circle of radius $a$ is $j_{\phi} \pi a^{2} d S$. In the present instance, $a$ is $r \sin \theta, d S$ is $r d r d \theta$, and we obtain

$$
\int j_{\phi} r \sin \theta d V=2 \mathscr{M} \text {. }
$$

The integral on the left-hand side of Eq. (II) may be expressed as a product of the dipole moment and a dimensionless parameter, $\xi$, which depends on the variation of $A_{\phi}$ throughout the star. If $\rho$ and $A_{\phi}$ are both constant throughout, $\xi$ is $3 / 4$, while if $A_{\phi}$ is proportional to $\mathrm{I} / r$, which it tends to be in the presence of strong axially symmetric convection, $\xi$ equals unity. Evidently $\xi$ does not vary enormously even for large changes in the spatial variation of $A_{\phi}$. Hence it follows that $d \mathscr{M} \mid \mathscr{M} d t$, the relative rate of decrease of the dipole moment $\mathscr{M}$ is about the same for a completely convective star as for a star in which no fluid motion occurs. A convective layer should have an even smaller effect on the magnetic decay time.

It must be emphasized that this result is based on an idealized model, and does not necessarily apply to actual stars. The analysis is restricted to two-dimensional motions, which are not, of course, to be anticipated. A spherical bounding surface is assumed with $\eta \rho$ constant up to the boundary, and a vacuum outside; quite apart from irregularities at the surface (a point emphasized informally by Sweet), an actual star is surrounded by ionized gas, and the concept of an 'external' field must be used with caution.

However, it is not physically obvious why more general motions should lead to a faster decay rate, since they would not appear to bend the lines of force more drastically than the restricted motions assumed here. In the lack of a more general approach, there seems to be no very strong reason at the moment to doubt that the dipole fields of the sun and the stars could well be fossil, i.e. residual magnetic fields present originally in the material from which the star condensed.

\section{THE NATURE OF MAgNETIG VARIABLE STARS}

The theoretical explanation of magnetic variables is certainly one of the most exciting and challenging problems in astrophysics. In addition to the large and unexpected variation of magnetic field, the great apparent 
variation of chemical composition with phase in the outstanding spectrum variables is not readily explained.

The first task of theory is, of course, to explain the observed variation of the magnetic field; and, in particular, the conspicuous reversal of this field in a number of spectrum variables. We believe that during the short time of one period-only a few days-the lines of force are frozen in to the fluid. Hence, if the same material is responsible for the absorption of the spectrum lines throughout the oscillation, this material must change its orientation by a large angle to account for the reversal of the magnetic field. No one has yet succeeded in thinking up any specific mechanism which would produce such a large change in orientation. A non-rotating magnetically pulsating star, of the type first analyzed by Schwarzschild [12], might produce small fluctuations in a large magnetic field. Such a configuration can account, perhaps, for some of the irregular magnetic variables-Babcock's Group I [13]—-but can apparently not produce the field reversals which are observed in about half of the magnetic variable stars.

We are thus forced to assume that at different phases of the outstanding magnetic variable stars we are looking at different regions, an assumption to which we would probably be forced in any case by the corresponding changes of chemical composition with phase. The simplest way in which a star can present different regions to the earth at different times is rotation, a mechanism which has been proposed for spectrum variables by A. Deutsch [14]. This is not the only way, however. One can imagine a non-rotating star whose surface is chiefly covered by two regions which have opposite magnetic polarity, and which oscillate in surface brightness. If the brightness oscillations are $180^{\circ}$ out of phase in the two regions, effects somewhat similar to those observed might be produced. Nonradial oscillations with large amplitudes might conceivably produce such effects; however, such a model apparently gives the wrong sign for the 'cross-over effect' observed by Babcock[15] in the line profiles. In the absence of supporting data this model will not be considered further.

Rotation of a star with a magnetic axis at an angle to the rotational axis is certainly the simplest explanation of magnetic variables, and should be explored first before more complicated models are involved. According to Deutsch [14] such a theory provides a quantitative explanation of the line profiles in terms of stellar rotation. In addition, rotation of an oblique rotator provides a very natural explanation of the 'cross-over effect'.

On the other hand, uniform rotation of some constant configuration is definitely not consistent with the observational data. In the magnetic 
stars which reverse polarity irregularly-Babcock's Group 2 [13]—no trace of a regular period has yet been found. Even in the spectrum variables, whose variations are, on the whole, periodic, the magnetic field changes are not strictly periodic. In $\alpha^{2} C V n$, for example, the changes of magnetic field strength observed by Babcock and Burd [16] are not sufficiently regular to be accounted for by solid body rotation. Also, the variations in radial velocity seem to be non-harmonic.

The peculiar abundances observed in all magnetic variables, and the particular changes observed in spectrum variables, pose serious problems. It is tempting to assume that such abundance anomalies are produced by nuclear reactions at the surface. Intense hydromagnetic disturbances might be expected to accelerate ions to very high energies, and the magnetic field may well inhibit convection, thus preventing the mixing of the peculiar surface material with the normal stellar matter deeper in the star. As pointed out by Babcock [13] this hypothesis provides another argument against uniform rotation of an unchanging configuration, since solid-body rotation of a magnetized fluid would not be expected to accelerate many charged particles.

It has not been pointed out that intense hydromagnetic oscillations may well be inevitable in a so-called oblique rotator. Let us consider a star in which the axis of the dipole field makes an angle with the angular momentum of the star. The magnetic forces will have an effect on the density distribution of the star. In the absence of a magnetic field the surfaces of equal density are oblate spheroids, symmetrical about the axis of rotation. A magnetic force will tend to distort these spheroids, and the star will no longer be symmetrical about its axis of rotation. As a result, the star will not be rotating about a principal axis of inertia.

A solid body, rotating about an axis that is not one of the principal axes of inertia, undergoes a wobbling motion, in which the instantaneous axis of rotation moves about, while the total angular momentum remains constant both in direction and magnitude. This type of motion is accompanied by stresses in the solid. In a magnetized fluid these stresses will give rise to distortions which vary in time, resulting, presumably, in hydromagnetic waves and magnetic oscillations. In the body of the star, where the magnetic forces are relatively small, such oscillations should be almost inappreciable, but near the surface, where the magnetic energy becomes comparable to the material energy density, large hydromagnetic oscillations may be possible. Such oscillations will dissipate energy, of course, and may be expected in time to bring the magnetic axis into coincidence with the axis of rotation. 
We are thus led to the following tentative picture of an idealized magnetic variable star. In a young star, newly formed from interstellar clouds, a fossil magnetic field will, in general, be present. The angle between the mean dipole field and the angular momentum will be arbitrary. In objects where the angle is appreciable, and where the rate of rotation is moderate, periodic changes of the magnetic field will be observed. Since the axis of rotation usually will not coincide with a principal axis of inertia, hydromagnetic oscillations will be superposed on the variations due to rotation. These oscillations may accelerate charged particles in the surface layers, and the transmutations produced by these particles may, perhaps, produce the differences in composition observed at the two magnetic poles. As the star grows older, the magnetic axis is brought into coincidence with the angular momentum vector, the magnetic variability and the hydromagnetic pulsations die away, fluid motions mix up the stellar material, eliminating the composition differences, and the star becomes more normal.

This working hypothesis can certainly not be regarded as established. It is presented to help stimulate further observations and additional theoretical work. Among the many questions raised by this hypothesis are the following. How can one account for Babcock's variable of Group 2, in which the polarity reverses irregularly? How can nuclear reactions produce different effects at the two magnetic poles? Why do differences in chemical composition occur in some magnetic variables, but are lacking in others, despite apparent similarities both in effective temperature and type of magnetic variation?

Certainly the answers to these questions will require modifications and changes in the simple hypothesis described above. The example of the solar atmosphere indicates that magnetic variations, both slow and rapid, may arise even when the axis of rotation agrees with the apparent dipole axis. It is evident that magnetic variable stars provide an exciting new field of astrophysics, whose detailed exploration and study has only just begun.

\section{REFERENCES}

[1] Cowling, T. G. Mon. Not. R. Astr. Soc. 113, 371, 1953.

[2] Elsasser, W. M. Amer. J. Phys. 23, 590, 1955.

[3] Elsasser, W. M. Amer. J. Phys. 24, 85, 1956.

[4] Elsasser, W. M. Rev. Mod. Phys. 28, I 35, 1956.

[5] Davis, L. and Greenstein, J. Astrophys. J. 114, 206, 195 I.

[6] Spitzer, L. and Tukey, J. Astophys. J. 114, 187, 1951.

[7] Piddington, J. H. p. 141 of this volume. 
[8] Mestel, L. and Spitzer, L. Mon. Not. R. Astr. Soc. 116, 503, 1956.

[9] Schwarzschild, M. Structure and Evolution of the Stars (Princeton University Press, 1958).

[1o] Cowling, T. G. Mon. Not. R. Astr. Soc. 94, 39, 1933.

[I I] Sweet, P. A. Mon. Not. R. Astr. Soc. 110, 69, 1950.

[12] Schwarzschild, M. Ann. Astrophys. 12, I48, 1949.

[13] Babcock, H. W. p, I6r of this volume.

[14] Deutsch, A. J. I.A.U. Transactions, vol. 8 (Cambridge University Press, 1954), p. 801 .

[15] Babcock, H. W. Astrophys. J. r14, I, I95I.

[16] Babcock, H. W. and Burd, S. Astrophys. J. 116, 8, 1952.

\section{Discussion}

Burbidge: Do you believe, in your picture of the formation of a protostar in the gas containing a magnetic field, that the magnetic field can help you to get rid of the excess angular momentum?

Spitzer: Yes, I believe that the field may remove angular momentum.

Burbidge: I have no idea how nuclear reactions could produce different abundance anomalies on different parts of the stellar surface. On the other hand, do you know of any mechanism which could separate elements on the surface after they are produced?

Spitzer: No.

Gold: The picture of the contracting protostar may be severely complicated by rotation. If there is a relative rotation within the mass of the star, perhaps enhanced by the initial contraction, then a twisting of the magnetic field may happen. The result of this is that the ionized gas on the lines of force may not be subjected to an outward force, as was supposed, but even to an inward one due to the spiralling of the lines which are wound around the region considered.

Spitzer: The angular velocity of rotation of the galaxy corresponds to a period of about $10^{8}$ years. The galaxy has to be contracted a lot more before it begins to twist up the field lines; I should think the contraction would take another Io $0^{5}$ years.

Gold: But in galaxies you have differential velocities of the order of $8 \mathrm{~km} / \mathrm{sec}$ and there will still be a possibility of twisting the field lines.

Spitzer: Did you state that the pressure will decrease?

Gold: Yes, if there is a twisting. I certainly agree that the main picture which you give has validity, but it may not be simple to deduce what the pressure is in case of a twisted magnetic field.

Spitzer: If the pressure actually decreases I think that the velocity would increase, but this cannot be true.

Gold: Well, in your case the force is directed outwards, but I do not think that your argument applies to the situation I am referring to.

Finally, I should like to point out that the problem of magnetic variable stars has some connexion with the investigation presented by Dr Davis the other day, i.e. the problem of the external flux from a simply connected body.

Schatzman: Is it possible to have orientation of interstellar particles by diamagnetic relaxation in the special case of graphite flakes? Cayrel and 
myself have shown that, due to their large optical and physical anisotropy, graphite flakes could well be the polarizing agent of interstellar space.

Spitzer: I do not think I can answer your question.

Schatzman: A difficulty for the formation of stars from protostars is the larger mass of the protostar if the protostar is to condense under its own weight. There is a way out of this difficulty. If the protostar is embedded in an $\mathbf{H}$ II region, the protostar can start contracting with a much smaller mass. In this connexion I would like to refer to the work of Ebert (Liège meeting, 1954) and to the beautiful photographs taken by Minkowsky which were shown at Cambridge in 1953. On these photographs, you can see several globules of small size, surrounded by the $H$ II region. If the $H$ II region pushes on the protostar, it can help it to contract considerably.

As you have brought here the explanation of many facts, would you not have also the hydromagnetic explanation of flare stars?

Spitzer: No, I do not think so.

Bostick: It is to be expected from the theoretical considerations by Kruskal and Schwarzschild that Taylor instability will set in when an ionized gas is being supported against a gravitational field by a magnetic field. This process is seen clearly in our experiments where plasmoids (essentially large-amplitude jets) projected almost radially inward across a magnetic field, spiral together to form a ring in the center. This process is obviously one where ionized matter can get from the outside to the inside across the magnetic field without carrying all the field lines along, and without having to wait for the plasma to diffuse across the lines. It should be pointed out that although in the laboratory experiments the central gravitational field is supplanted by inertial fields produced by the deceleration of the plasmoids, the resultant plasma ring has a certain amount of stability and furthermore has angular momentum and very probably a magnetic moment. Although as yet, no scaling factors from laboratory conditions to cosmical conditions have been given thorough consideration, it is nevertheless worth while to consider the effect of this Taylor instability process in starproduction in regions where $\mathrm{H}$ II represents any appreciable fraction of the $\mathrm{H}_{\mathbf{I}}$ present.

Cowling: May I ask if the discussion of the second problem depended essentially on the assumption of incompressibility? I ask this in view of Walén's suggestion that as a consequence of compressibility, convection in the sun's surface layers might actually carry lines of force down below the solar surface.

Spitzer: The results for the sphere were derived for the compressible case.

Cowling: Is your second problem essentially a two-dimensional one?

Spitzer: Yes.

Mestel: The process discussed by Dr Spitzer and myself, by which the bulk of the contracting protostar can slip across the lines of force, is closely related to the 'ambipolar diffusion' of Dr Schlüter. The importance of this has recently been emphasized by Dr Piddington and Professor Cowling. We differ in that the drift of plasma through the neutral gas is not restricted to be small compared with the motion of the gas as a whole.

There is one aspect of the process which should be underlined. What is being destroyed by collisions between plasma and neutral gas is magnetic energy, not 
magnetic flux. If the ohmic field and the partial pressures are ignored, the magnetic field is frozen into the plasma; the motion of the system 'field plus plasma' is determined by the balance between the magnetic force and the friction between neutral gas and plasma. All the theorems of ordinary hydromagnetics which follow from the identical motion of matter and magnetic field may be taken over by substituting the velocity of the plasma for the velocity of the gas as a whole. Thus, if we wish to destroy magnetic flux, we must employ ohmic dissipation to bring about relative motion of field and plasma. 